\title{
Representing Diffusion MRI in 5-D Simplifies Regularization and Segmentation of White Matter Tracts
}

\author{
Lisa Jonasson*, Xavier Bresson, Jean-Philippe Thiran, Van J. Wedeen, and Patric Hagmann
}

\begin{abstract}
We present a new five-dimensional (5-D) space representation of diffusion magnetic resonance imaging (dMRI) of high angular resolution. This 5-D space is basically a non-Euclidean space of position and orientation in which crossing fiber tracts can be clearly disentangled, that cannot be separated in three-dimensional position space. This new representation provides many possibilities for processing and analysis since classical methods for scalar images can be extended to higher dimensions even if the spaces are not Euclidean. In this paper, we show examples of how regularization and segmentation of $d M R I$ is simplified with this new representation. The regularization is used with the purpose of denoising and but also to facilitate the segmentation task by using several scales, each scale representing a different level of resolution. We implement in five dimensions the Chan-Vese method combined with active contours without edges for the segmentation and the total variation functional for the regularization. The purpose of this paper is to explore the possibility of segmenting white matter structures directly as entirely separated bundles in this 5-D space. We will present results from a synthetic model and results on real data of a human brain acquired with diffusion spectrum magnetic resonance imaging (MRI), one of the dMRI of high angular resolution available. These results will lead us to the conclusion that this new high-dimensional representation indeed simplifies the problem of segmentation and regularization.
\end{abstract}

Index Terms-diffusion magnetic resonance imaging (MRI), five dimensional level sets, white matter segmentation and position orientation space.

\section{INTRODUCTION}

D IFFUSION magnetic resonance imaging (dMRI) is a modality that permits noninvasive quantification of water diffusion in living tissues. The tissue structure affects the Brownian motion of the water molecules, which leads to an anisotropic diffusion. Today, a diffusion tensor (DT) model [1],

Manuscript received October 15, 2006; revised April 17, 2007. This work was supported by the National Institutes of Health under Grant 1R01-MH64044 and the Swiss National Science Foundation under Grant 2153-066943.01 and Grant 3235B0-102863. Asterisk indicates corresponding author.

*L. Jonasson, is with the Signal Processing Institute (ITS), École Polytechnique Fédérale de Lausanne (EPFL), CH-1015 Lausanne, Switzerland (e-mail: lisa.jonasson@gmail.com)

X. Bresson, J.-P. Thiran, and P. Hagmann are with the Signal Processing Institute (ITS), École Polytechnique Fédérale de Lausanne (EPFL), CH-1015 Lausanne, Switzerland.

V. J. Wedeen is with the Athinoula A. Martinos Center for Biomedical Imaging, Massachusetts General Hospital and the Harvard Medical School, Boston, MA 02115 USA.

P. Hagmann is with the Department of Radiology, Lausanne University Hospital (CHUV), CH-1011 Lausanne, Switzerland.

Color versions of one or more of the figures in this paper are available online at http://ieeexplore.ieee.org.

Digital Object Identifier 10.1109/TMI.2007.899168
[2] is the most frequently used method to map the structural anisotropy. The tensor model, which basically only contains information about anisotropy and principal diffusion direction, has limited capabilities of resolving complex brain white matter architectures, particularly in regions with fiber crossings [3].

A recent approach, first presented by Wedeen et al. in [4], and fully developed in [5], is diffusion spectrum imaging (DSI). This technique is based on the q-space theory first presented by Callaghan [6] in 1990 and further developed by Cory and Garroway [7]. Wedeen et al. demonstrated that the q-space approach is feasible in vivo and can be used to resolve complex fiber orientation by construction of the a full 3-D probability density function (PDF) of the diffusion at each location [5]. This PDF provides a detailed description of the diffusion and manages to resolve complex tissue architecture such as fiber crossings [8] For simplicity, the PDF is normally reduced to an orientation density function (ODF), which is the radial projection of the PDF. Other approaches such as $q$-ball imaging [9], spherical deconvolution [10], and persistent angular structure (PAS) [11] aim at directly obtaining the ODF without first measuring the PDF.

The brain white matter is made of nerve fibers which are organized in bundles or tracts-sets of fibers that have close endpoints and similar trajectories. The process of fiber tractography aims at inferring fiber trajectories by computing lines of maximal diffusion coherence through the data. By appropriate selection, bundles of interest can be visualized [12]. Such tractography methods are in general used to map cerebral connectivity throughout the brain [13], [14]. However a fiber tract is not only a set of lines but can also be considered as a single object that one may want to identify, in order to analyze its shape or to measure some intrinsic properties. Jonasson et al. [15] presented a 3-D geometric flow algorithm designed for segmenting fiber tracts as regions in a DT-MRI data, which is however limited in areas of fiber crossings because of the limited angular resolution of DT-MRI.

In many image processing problems the goal is often to reduce the dimensionality of the data, but sometimes it can actually be useful to augment it. Hagmann et al. [16] proposed to model dMRI data as a scalar field on a discrete five-dimensional (5-D) space and use a Markov random field segmentation methodology to identify fiber tracts in the brain [16]. Following this early work, we discuss a level set based technique implemented on a continuous 5-D space for segmenting and regularizing fiber tracts from dMRI data of high angular resolution. The original contribution of our paper is mainly the exploration of the possibility of segmenting white matter structures directly 
as separate bundles in 5-D space and the implementation of the well known segmentation method, total variation (TV) flow, in a 5-D space.

\section{BACKGROUND THEORY}

First, we explain how position-orientation space (POS)—our 5-D space-is mathematically constructed and how it can be obtained from the dMRI data. We then explain the regularization and segmentation techniques used. They are based on classical partial differential equations (PDE) in $N$-dimensions. The method used for the segmentation is the Chan-Vese approach, "Active contours without edges" [17] implemented using the level set method proposed by Osher and Sethian [18]-[20]. The level set formalism is defined for $N$-dimensions and we show how to practically apply it in a 5-D non-Euclidean space, with an emphasis on the computation of the mean curvature in five dimensions for contour regularization. The regularization is used both for denoising and to link the scales in a multiresolution approach and is done by the TV flow [21], [22].

\section{A. Position Orientation Space}

A diffusion MRI experiment of high angular resolution (such as DSI [5], Q-ball [23], spherical deconvolution [10], and PAS [11]) provides a 3-D map of ODFs. Thus, for every position vector $(x, y, z)$ in Euclidean 3-D space, namely $\mathbb{R}^{3}$, there is an ODF measuring the intensity of diffusion in any orientation $(\varphi, \theta)$ restricted to the unit sphere, namely $\mathbb{S}^{2}$, with the azimuthal angle $\theta \in[0,2 \pi)$ and the polar angle $\varphi \in[0, \pi]$. The POS, called $\Omega$, is defined by the direct Cartesian product of the vector spaces $\mathbb{R}^{3}$ and $\mathbb{S}^{2}$ such that a non-negative scalar function $I$ defined in the POS is as follows:

$$
\begin{aligned}
I: \quad \Omega & =\mathbb{R}^{3} \times \mathbb{S}^{2} \rightarrow \mathbb{R}^{+}, \\
\omega & =(x, y, z, \varphi, \theta) \rightarrow I(\omega) .
\end{aligned}
$$

This new vector space comes equipped with a metric, namely $g_{\Omega}$, derived from a tensor direct sum such as

$$
g_{\Omega}=g_{\mathbb{R}^{3}} \oplus g_{\mathbb{S}^{2}}
$$

also expressed by

$$
g_{\Omega}=\operatorname{diag}\left(g_{\mathbb{R}^{3}}, g_{\mathbb{S}^{2}}\right),
$$

where the matrix $\operatorname{diag}\left(\Lambda_{1}, \ldots, \Lambda_{k}\right)$ is a diagonal matrix in which the diagonal is given by $\left(\Lambda_{1}, \ldots, \Lambda_{k}\right)$ where $\Lambda_{1 \leq l \leq k}$ can be either scalar or matrix, the standard Euclidean metric is given by $g_{\mathbb{R}^{3}}(x, y, z)=\left(\delta_{i j}\right)$ whose $\delta_{i j}$ is the Kronecker function and $1 \leq i, j \leq 3$ are the indices of the tensor, and the standard spherical metric is provided by $g_{\mathbb{S}^{2}}(\varphi, \theta)=\operatorname{diag}\left(1, \sin ^{2} \varphi\right)$. The metric tensor (2) of the POS allows to compute the distance between any two points on the manifold $\Omega$. Indeed, in a Riemannian manifold, the length of segment of a curve parametrized by $p$, from $p_{1}$ to $p_{2}$, is defined by the formula (given in, e.g., [24]) $\int_{p_{1}}^{p_{2}} \sqrt{\sum_{i, j=1}^{5}\left(g_{\Omega}\right)_{i j}\left(d \omega_{i} / d p\left(d \omega_{j} / d p\right)\right.} d p,\left(g_{\Omega}\right)_{i j}$ is the $(i, j)$ th component of the tensor $g_{\Omega}, \omega_{1 \leq i \leq 5}, \omega_{1 \leq j \leq 5} \in\{x, y, z, \varphi, \theta\}$.

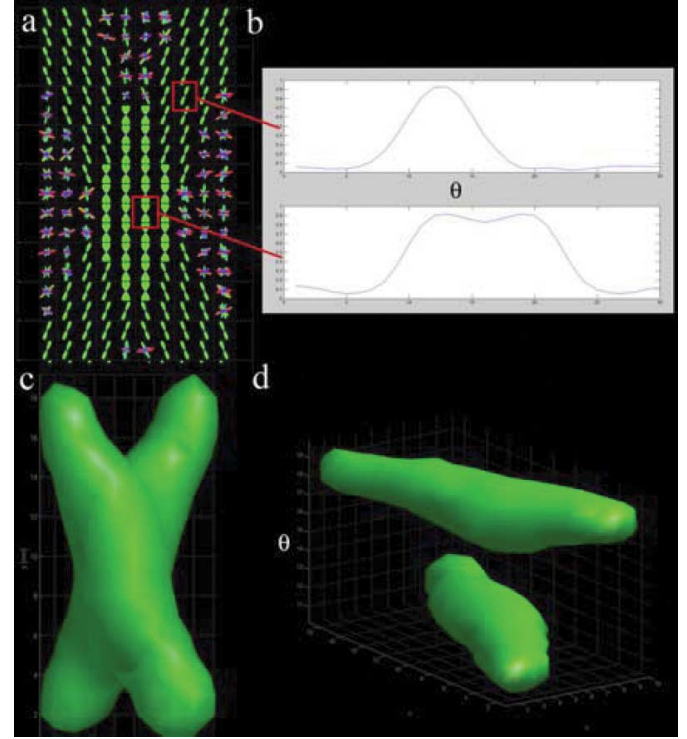

Fig. 1. Example of POS for a 2-D slice of a volume of ODFs. (a) The 2-D field of ODFs. (b) Intensity is plotted for each angle, $\theta$. (c) If the angle, $\theta$, is not considered the crossing fibers cannot be separated. (d) Angle $\theta$ constitutes the third dimension of the space and the value in each position is the intensity, I. Hence, in three dimensions the two fibers can be segmented as two separate structures.

It also provides the gradient operator defined on this non-Euclidean space that will be useful later

$$
\nabla_{\Omega}=\sqrt{\left(g_{\Omega}\right)^{-T}} \nabla_{\mathbb{R}^{5}}
$$

which simplifies to

$$
\nabla_{\Omega}=e_{x} \frac{\partial}{\partial x}+e_{y} \frac{\partial}{\partial y}+e_{z} \frac{\partial}{\partial z}+e_{\varphi} \frac{\partial}{\partial \varphi}+e_{\theta} \frac{1}{\sin \varphi} \frac{\partial}{\partial \theta}
$$

given a 5-D standard basis $\left(e_{x}, e_{y}, e_{z}, e_{\varphi}, e_{\theta}\right)$ and the operator $(\cdot)^{-T}$ as the inverse tensor transpose.

To get some intuition about what POS is and why it is useful for fiber tract segmentation, it is instructive to consider the case of a 2-D map of ODF restricted to a plane. In Fig. 1(a), a 2-D slice of ODFs is shown. The slice shows a crossing between two fiber tracts. The ODFs in the figure are restricted to the plane and can therefore be described through only one angle, $\theta$. The intensity of the ODF varies with the angle. In the case where we only have one fiber there will be one peak intensity for the angle that corresponds to the direction of the fiber. In positions where two fiber tracts cross there will be two intensity peaks, one for the direction of each fiber. These two cases are illustrated in Fig. 1(b).

The third dimension, $\theta$, represents the orientation of diffusion; hence, the 2-D ODF map is mapped as a 3-D scalar field. This means that even though the two fiber tracts cross over in 2-D, they will be separated in 3-D and can therefore easily be segmented as two independent structures. Fig. 1(d) shows how the two fiber tracts that cannot be separated in 2-D [Fig. 1(c)] can be segmented in 3-D where the angle, $\theta$, constitutes the third dimension. In Fig. 2, an example of a slice from a 3-D volume of ODFs is shown. The ODFs are here described by two angles 


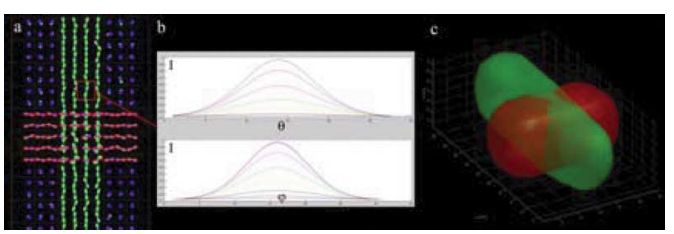

Fig. 2. (a) Slice of a synthetic 3-D volume of ODFs. (b) The intensity, $I$, of the different angles, $(\theta, \varphi)$, plotted against each other. (c) The synthetic fiber tracts have been segmented in 5-D and projected back into 3-D.

and the segmentation is done in 5-D and then projected back in 3-D for visualization.

\section{B. Segmentation in the Position Orientation Space}

1) Level Set Method in the N-D Euclidean Space: Since the level set method was introduced by Osher and Sethian [18]-[20], it has become a more and more popular analytical and numerical tool for image processing, fluid mechanics, graphics, computer vision, etc. It is basically used for tracking moving fronts by considering the front as the zero level set of an embedding function, called the level set function. In image processing, the level set method is most frequently used as a segmentation tool through propagation of a contour by using the properties of the image as well as properties of the contour itself, such as the mean curvature. It was originally used to detect edges in an image [25], [26], but more recent applications detect textures, shapes, colors, etc. One of the main advantages of the level set theory is its natural extension to $N$-dimensional images, which is usually difficult to achieve with standard segmentation models. Thus, the extension to three-dimensional images is commonly used, e.g., [27], and even though some of the properties of the 2-D curves, such as the property of shrinking to a point under curvature flow, do not hold in the 3-D case, the main part of the theory remains valid and works well for segmentation of 3-D objects. The extension to even higher dimensions is straightforward, which will allow us to use the level set method in the 5-D POS.

In the rest of this section, we introduce the basics of the level set method in the $N$-D Euclidean space. Let the level set function, $\phi(\mathbf{x}, t)$, be a smooth function where $\mathbf{x}=\left(x_{1}, \ldots, x_{N}\right) \in$ $\mathbb{R}^{N}, t \in \mathbb{R}^{+}$. The $(N-1)$-D hypersurface in $N$-dimensions is represented by the zero level set of $\phi$ such that $S(t)=\{\mathbf{x} \in$ $\left.\mathbb{R}^{N}: \phi(\mathbf{x}, t)=0\right\}$. Then the evolution of the hypersurface, embedded in the level set function, is described through the following general partial differential equation (PDE) in any dimension [18]-[20]:

$$
\frac{\partial \phi}{\partial t}=F|\nabla \phi|
$$

where $F$ is a speed function depending on the given application.

A fundamental task in the level set framework is the regularization/smoothing of the moving interface to get satisfactory segmentation results. For this particular case, the speed function is usually based on the mean curvature $\kappa$ whose general expression in $N$-D is as follows:

$$
F=-\kappa=-\nabla \cdot\left(\frac{\nabla \phi}{|\nabla \phi|}\right) \text {. }
$$

For this particular function $F$, (5) becomes the well-known Euclidean mean curvature flow or Euclidean shortening flow. In 2-D, the developed expression of the mean curvature is as follows:

$$
\nabla \cdot\left(\frac{\nabla \phi}{|\nabla \phi|}\right)=\frac{\phi_{x x} \phi_{y}^{2}-2 \phi_{x} \phi_{y} \phi_{x y}+\phi_{y y} \phi_{y}^{2}}{\left(\phi_{x}^{2}+\phi_{y}^{2}\right)^{1 / 2}} .
$$

The expression of the mean curvature is naturally longer in 3-D and even more in higher dimensions. For example, computing the curvature formula for 5-D, Mathematica gives a several-pages-long answer that is obviously not satisfactory from a numerical point of view. Fortunately, a lot of work has already been done for $N$-D mean curvature flows [28], [29]. Hence, we propose to use the theory developed by Ambrosio and Soner [28] to determine the mean curvature of a $(N-1)$-D hypersurface embedded in the Euclidean $N$-D space.

As explained in, e.g., [24], the mean curvature of a smooth hypersurface, $S$, is described by the mean of the principal curvatures $\left\{\kappa_{i}\right\}_{1 \leq i \leq N-1}$ of $S$ as follows:

$$
\kappa=\frac{\kappa_{1}+\cdots+\kappa_{N-1}}{N-1} .
$$

In [28], Ambrosio and Soner showed that the $N-1$ principal curvatures of a hypersurface $S$, represented by the zero level set of a function $\phi: \mathbb{R}^{N} \rightarrow \mathbb{R}^{+}$such that $S=\left\{\mathbf{x} \in \mathbb{R}^{N}: \phi(\mathbf{x})=\right.$ $0\}$, are given by the $N-1$ smallest eigenvalues of the following $N \times N$ symmetric matrix:

$$
J(\mathbf{x})=\frac{1}{|\nabla \phi(\mathbf{x})|^{2}} P_{\nabla \phi(\mathbf{x})} \nabla^{2} \phi(\mathbf{x}) P_{\nabla \phi(\mathbf{x})}
$$

where $\nabla^{2}$ is the $N \times N$ Hessian matrix and $P_{p}$ is a projection operator onto the space normal to the nonzero vector $p \in \mathbb{R}^{N}$ defined as

$$
P_{p}=1-\frac{p \otimes p}{|p|^{2}}
$$

where 1 is the identity matrix and $\otimes$ is the tensor product. Hence, the practical calculation of the mean curvature of a $(N-1)$-D hypersurface is numerically done by first computing the matrix $J$ then determining its eigenvalues.

We now introduce the efficient image segmentation model of Chan and Vese for $N$-D scalar images. The model of Chan and Vese presented in [17] is a method for segmenting scalar images based on homogenous region detection by using the weak formulation of the influential Mumford and Shah functional [30]. The variational model of Chan and Vese is as follows:

$$
\begin{aligned}
\min _{S_{\mathrm{in}}, c_{\mathrm{in}}, c_{\text {out }}} & \left\{E_{\mathrm{CV}}\left(S_{\mathrm{in}}, c_{\mathrm{in}}, c_{\mathrm{out}}, \lambda\right)\right. \\
= & \operatorname{Per}\left(S_{\mathrm{in}}\right)+\lambda \int_{S_{\mathrm{in}}}\left(c_{\mathrm{in}}-u(\mathbf{x})\right)^{2} d \mathbf{x} \\
& \left.+\lambda \int_{\mathbb{R}^{N} \backslash S_{\text {in }}}\left(c_{\text {out }}-u(\mathbf{x})\right)^{2} d \mathbf{x}\right\}
\end{aligned}
$$

where $u$ is a given scalar image, $S_{\text {in }}$ is a closed subset of the image domain $\mathbb{R}^{N}$ whose boundary is the hypersurface $S$, $\operatorname{Per}\left(S_{\text {in }}\right)$ is the perimeter of the set $S_{\text {in }}$, i.e., the hyper-area of 
the hypersurface $S, \lambda$ is an arbitrary positive parameter which controls the tradeoff between the regularization process and the fidelity of the solution with respect to the original image $u$, constants $c_{\text {in }}, c_{\text {out }}$ are in $\mathbb{R}$, and $d \mathbf{x}=d x_{1} \ldots d x_{N}$ is the infinitesimal invariant volume in the $N$-D Euclidean space. The variational model (11) determines the best approximation, in the $L^{2}$-sense, of the image $u$ as a set of (nonconnected) regions with only two different values $c_{\text {in }}$ and $c_{\text {out }}$. As noticed in [17], if $S_{\text {in }}$ is fixed, the values $c_{\text {in }}$ and $c_{\text {out }}$ which minimize the energy $E_{\mathrm{CV}}$ are the mean intensity values inside and outside $S$. Finally, the term $\operatorname{Per}\left(S_{\text {in }}\right)$ imposes a smoothness constraint on the geometry of the set $S_{\text {in }}$, i.e., the hypersurface $S$, which separates the piecewise constant regions. Chan and Vese represent the regions $S_{\text {in }}$ and its complementary $\mathbb{R}^{N} \backslash S_{\text {in }}$ with the Heaviside function, $H$, of a level set function $\phi$ as follows:

$$
\begin{aligned}
& E_{\mathrm{CV}}^{2}\left(\phi, c_{\mathrm{in}}, c_{\mathrm{out}}, \lambda\right) \\
& =\int_{\mathbb{R}^{N}}|\nabla H(\phi(\mathbf{x}))| d \mathbf{x}+\lambda \int_{\mathbb{R}^{N}}\left[H(\phi(\mathbf{x}))\left(c_{\mathrm{in}}-u(\mathbf{x})\right)^{2}\right. \\
& \left.\quad+H(-\phi(\mathbf{x}))\left(c_{\mathrm{out}}-u(\mathbf{x})\right)^{2}\right] d \mathbf{x} .
\end{aligned}
$$

The flow minimizing Energy $E_{\mathrm{CV}}^{2}$ with respect to $\phi$ is the following:

$$
\begin{aligned}
& \frac{\partial \phi}{\partial t}(\mathbf{x}, t)=\delta(\phi(\mathbf{x}, t)) {\left[\nabla \cdot\left(\frac{\nabla \phi(\mathbf{x}, t)}{|\nabla \phi(\mathbf{x}, t)|}\right)\right.} \\
&\left.-\lambda\left(c_{\mathrm{in}}-u(\mathbf{x})\right)^{2}+\lambda\left(c_{\mathrm{out}}-u(\mathbf{x})\right)^{2}\right]
\end{aligned}
$$

where $\delta$ is the Dirac function. As we said, $c_{\text {in }}$ and $c_{\text {out }}$ which minimize Energy $E_{\mathrm{CV}}^{2}$ are respectively the intensity averages in the regions $S_{\text {in }}=\left\{\mathrm{x} \in \mathbb{R}^{N}: \phi(\mathrm{x}, t)>0\right\}$ and $\mathbb{R}^{N} \backslash S_{\text {in }}=$ $\left\{\mathbf{x} \in \mathbb{R}^{N}: \phi(\mathbf{x}, t)<0\right\}$ such that

$$
\left\{\begin{array}{l}
c_{\mathrm{in}}=\frac{\int_{\mathbb{R}^{N}} H(\phi(\mathbf{x})) u(\mathbf{x}) d \mathbf{x}}{\int_{\mathbb{R}^{N}} H(\phi(\mathbf{x})) d \mathbf{x}} \\
c_{\text {out }}=\frac{\int_{\mathbb{R}^{N}}(H(-\phi(\mathbf{x}))) u(\mathbf{x}) d \mathbf{x}}{\int_{\mathbb{R}^{N}}(H(-\phi(\mathbf{x}))) d \mathbf{x}}
\end{array} .\right.
$$

2) Level Set Method in the POS: In this section, we apply the previous theory defined in the $N$-D Euclidean space to the POS by taking the geometric counterparts such as the gradient, the curvature, and the intensity average defined in the POS.

Let a level set function $\phi(\omega, t)$, where $\omega=(x, y, z, \varphi, \theta) \in \Omega$ as defined in (1) and $t \in \mathbb{R}^{+}$, be a smooth function defined in the 5-D POS. Then a 4-D hypersurface is represented by the zero level set of $\phi$ such that $S(t):=\{\omega \in \Omega: \phi(\omega, t)=0\}$.

The counterpart of the segmentation model of Chan and Vese [17] defined in (13) is as follows:

$$
\begin{aligned}
& \frac{\partial \phi}{\partial t}(\omega, t)=\delta(\phi(\omega, t)) {\left[\nabla_{\Omega} \cdot\left(\frac{\nabla_{\Omega} \phi(\omega, t)}{\left|\nabla_{\Omega} \phi(\omega, t)\right|}\right)\right.} \\
&\left.-\lambda\left(c_{\mathrm{in}}^{\Omega}-I(\omega)\right)^{2}+\lambda\left(c_{\mathrm{out}}^{\Omega}-I(\omega)\right)^{2}\right]
\end{aligned}
$$

where $I(\omega)$ is a given scalar image in the POS, $\nabla_{\Omega}$ is the gradient operator in the non-Euclidean POS defined in (3), $c_{\mathrm{in}}^{\Omega}, c_{\mathrm{out}}^{\Omega}$ are the intensity averages in the regions $S_{\text {in }}=\{\omega \in \Omega$ :

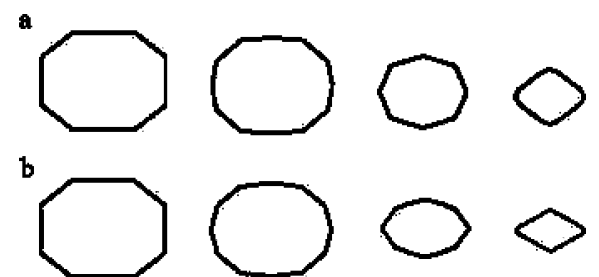

Fig. 3. Hyper-cube evolving under 5-D mean curvature flow. (a) $x-z$-plane. (b) $y-\theta$-plane.

$\phi(\omega, t)>0\}$ and $\Omega \backslash S_{\text {in }}=\{\omega \in \Omega: \phi(\omega, t)<0\}$ taking into account the non-Euclidean geometry of the POS such that

$$
\left\{\begin{array}{l}
c_{\mathrm{in}}^{\Omega}=\frac{\int_{\Omega} H(\phi(\omega)) I(\omega) d \omega}{\int_{\Omega} H(\phi(\omega)) d \omega} \\
c_{\mathrm{out}}^{\Omega}=\frac{\int_{\Omega}(H(-\phi(\omega))) I(\omega) d \omega}{\int_{\Omega}(H(-\phi(\omega))) d \omega}
\end{array}\right.
$$

where $d \omega=\sin \varphi d x d y d z d \varphi d \theta$ is the infinitesimal invariant volume in the POS. The term $\nabla_{\Omega} \cdot\left(\nabla_{\Omega} \phi /\left|\nabla_{\Omega} \phi\right|\right)$ in (15) plays the role of the mean curvature of the level sets of $\phi$ in the non-Euclidean POS. It is computed by strict analogy of the approach proposed by Ambrosio and Soner [28] described in the previous section. Thus, the mean curvature of the 4-D surface embedded in the 5-D POS is the average value of the four smallest eigenvalues of the following $5 \times 5$ symmetric matrix:

$$
J(\omega)=\frac{1}{\left|\nabla_{\Omega} \phi(\omega)\right|^{2}} P_{\nabla_{\Omega} \phi(\omega)} \nabla_{\Omega}^{2} \phi(\omega) P_{\nabla_{\Omega} \phi(\omega)} .
$$

We have tested the curvature term of (15) on a synthetic surface by evolving a 5-D hyper-cube through the mean curvature flow and seen how it first turns into a hyper-sphere and then finally shrinks to a point; see Fig. 3 .

We have defined in this section the level set-based segmentation model. Once the POS was defined in Section II-A, we have seen that a scalar image in the POS is not too different from a classical gray-scale image. The specific considerations except for the high number of dimensions are the periodicity of the angles $(\varphi, \theta)$ and the computations of the gradient, the curvature and the intensity average defined in the POS. From a theoretical point of view, the PDE (15) looks straightforward to implement but is practically difficult. One of the main problems is handling the storage of the huge amount of data that is treated. Optimizing the computation of the level set function and its reinitialization are thus crucial.

\section{Multiresolution Segmentation Using Total Variation-Flow}

The 5-D POS contains a multitude of fibers and it is hard to determine an appropriate initialization for the segmentation. By using a multiresolution segmentation approach [31], [32], we reduce the number of possible fiber tracts to segment and facilitate the finding of the major tracts. The segmentation is therefore first done on a highly smoothed image to catch the main shape of the tract. The segmentation is then successively refined by augmenting the resolution of the 5-D space. A well-known problem with the level set method is that the 
solution is always a local minimum. By performing the segmentation task at different scales, the multiresolution approach avoids many nonoptimal minima. The resolution reduction is performed scale by scale with anisotropic filtering/diffusion, a method that smoothes the image while keeping the edges. Many anisotropic diffusion methods have been suggested in the literature; for a review, we refer to [33]. We have chosen the total variation (TV) flow to perform the multiscale regularization in the POS.

The idea of minimizing the total variation norm of a function $u \in L^{1}\left(\mathbb{R}^{N}\right)$

$$
T V(u)=\int_{\mathbb{R}^{N}}|\nabla u(\mathbf{x})| d \mathbf{x}
$$

for image processing purposes was first introduced by Rudin, Osher, and Fatemi in [21]. This functional does not penalize discontinuities so the image is smoothed while keeping the original edges. The standard minimization of the TV-flow is done by the gradient descent method which yields the following equation [22]:

$$
\frac{\partial u}{\partial t}(\mathbf{x}, t)=\nabla \cdot\left(\frac{\nabla u(\mathbf{x}, t)}{|\nabla u(\mathbf{x}, t)|}\right)
$$

where $t \in \mathbb{R}^{+}$is introduced as usual. Here, $u(\mathbf{x}, t)$ is what we call the multiscale/multiresolution image. A multiscale image is a representation at a continuum of scales $t$, embedding the original image $u(\mathbf{x}, t=0)$ into a family $u(\mathbf{x}, t>0)$ of gradually smoother and simplified versions. As in the previous section, we define the TV-flow in the POS by strict analogy with the Euclidean case. The TV-flow in the POS is thus defined by

$$
\frac{\partial I}{\partial t}(\omega, t)=\nabla_{\Omega} \cdot\left(\frac{\nabla_{\Omega} I(\omega, t)}{\left|\nabla_{\Omega} I(\omega, t)\right|}\right)
$$

with $I(\omega, t):\left(\Omega, \mathbb{R}^{+}\right) \rightarrow \mathbb{R}^{+}$.

\section{Method AND IMPLEMENTATION}

\section{A. Creating POS}

We construct the 5-D POS from a 3-D map of ODF. The data is stored in a 5-D matrix where the first 3-D code for the location and the last two dimensions for the orientation. The spherical geometry of the space in the ODF yields a periodicity in the data. Because the ODF is a symmetric function only a hemisphere of the ODF in each location needs to be sampled, i.e., only half of POS needs to be stored. Accordingly, the values of the half ODF are placed on a 2-D grid of following orientation:

$$
(\varphi, \theta) \in\left\{0, \frac{\pi}{n}, \ldots, \pi-\frac{\pi}{n}\right\} \times\left\{0, \frac{\pi}{n}, \ldots, \pi-\frac{\pi}{n}\right\}
$$

where $n$ is the sampling step. In practice, the sampling step, $n$, has been chosen empirically in order to provide a sufficiently high resolution so that crossing fibers will be clearly separated while keeping orientation space sufficiently small for computational purposes (here $n=18$ ).

Thanks to the symmetry of the data, we work with only half the data, but this involves that we need to avoid border artifacts by exchanging the two ends of the level set along the $\theta$-axis after every iteration.

\section{B. Reducing the Segmentation Space}

Even though the problem of crossing fibers are solved with our approach, a problem that is not avoided when segmenting the fiber tracts is the well-known problem of kissing fibers. Kissing fibers are when two fiber tracts at one point share both position and orientation. If no external interference is added the kissing tract will be segmented as one unit. This phenomenon can complicate the separation of two tracts and to avoid this problem a priori knowledge about the structures was used to remove parts of the orientation-space corresponding to angles that are not present in the structures to be segmented. This operation does not only separate tracts but it also speeds up the convergence and the processing times by reducing the space.

\section{Solving the Level Set Equation}

The level set equation is solved with a classical upwind-scheme, see, e.g., [20]. As it is usually done, the level set function $\phi$ is implemented by the signed distance function (SDF) of the evolving surface. Due to local dependence of the propagation speed, the evolution of the other level sets differs from the zero level set. This creates irregularities that deform $\phi$ so it ceases to be a signed distance function. A correct SDF is crucial to get a correct and smooth evolution of the surface, since the calculations of the normals and curvatures depend directly on the SDF. Therefore, a reinitialization of the signed distance map is made at every iteration. It is implemented using the fast marching method to solve the following PDE [34]:

$$
|\nabla \phi|=1 \text {. }
$$

During the evolution process it is very important to preserve a SDF to assure a correct computation of the normals and the mean curvature. If we do not reinitialize the level set function the propagation quickly becomes unstable. The step length of the iteration also needs to be sufficiently small to maintain the smoothness of the propagating surface. We have chosen a maximum step length of $10 \%$ of the voxel size. The iterations are continued until the total movement of the propagating surface is considered insignificant.

To speed up this process, all values above 4 of the SDF is set to 4 . The reinitialization process is then only performed until the value 4 is reached.

\section{Projection in the Position Space}

The proposed segmentation method has been defined in the 5-D position orientation space. Hence, the solution lies in a 5-D space, which obviously cannot be vizualized. Thus, the segmentation result has to be projected in the 3-D position space by carefully extracting the orientation information. The projection back to the 3 -D position space $\mathbb{R}^{3}$ is very simple. The value of the level set function in $\mathbb{R}^{3}$, called $\phi_{\mathbb{R}^{3}}$, is the maximum of the values of the level set function $\phi$ in the orientation space

$$
\phi_{\mathbb{R}^{3}}(x, y, z)=\max _{\varphi, \theta} \phi(x, y, z, \varphi, \theta)
$$

if the inside region $S_{\text {in }}$ of the hypersurface $S$ is defined by $\{\omega \in$ $\Omega: \phi(\omega, t)>0\}$ and the outside region $\Omega \backslash S_{\text {in }}$ by $\{\omega \in$ $\Omega: \phi(\omega, t)<0\}$. We point out that the operator max in (23) is replaced by $\mathrm{min}$ if the inside region $S_{\mathrm{in}}$ is represented by 


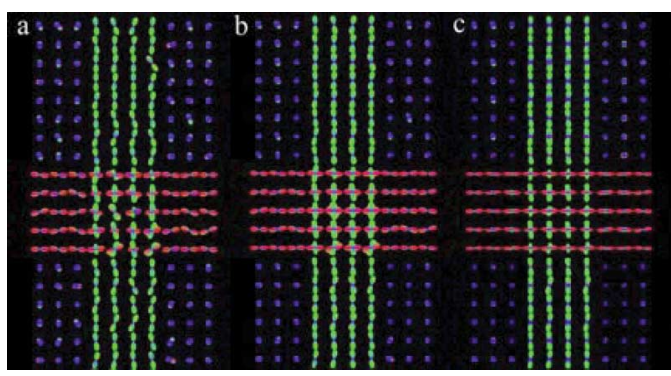

Fig. 4. A 2-D section of the 3-D space of the ODF volumes at different scales. (a) No smoothing applied. (b) Smoothed with a TV-flow, ten iterations. (c) Twenty iterations.

$\{\omega \in \Omega: \phi(\omega, t)<0\}$ and the outside region $\Omega \backslash S_{\text {in }}$ by $\{\omega \in \Omega: \phi(\omega, t)>0\}$. Finally, the surface representing the segmented structures is given by

$$
S_{\mathbb{R}^{3}}=\left\{(x, y, z) \in \mathbb{R}^{3}: \phi_{\mathbb{R}^{3}}(x, y, z)=0\right\} .
$$

One way to understand (23) is to observe Fig. 1. If $(x, y)$ is located inside a fiber in the 2-D position space, Fig. 1(c), then the maximum value along the $\theta$-axis, Fig. 1(d), will be positive. Inversely, if $(x, y)$ is outside a fiber then the maximum value will be negative. The contour of the fiber naturally lies on the boundary between positive and negative values.

\section{RESULTS}

\section{A. Synthetic Data}

To test the method, we constructed a 3-D map of ODFs modelling two crossing fiber tracts; see left figure in Fig. 4. The ODFs are normalized by dividing with the maximum of each ODF. In Fig. 4, we see the effect of the regularization through the TV flow with 10 and 20 iterations.

To perform the segmentation the hyper-surfaces were initialized by placing a small hyper-surface of a few 5-D voxels in each fiber tract. The hyper surface was evolved until convergence and then projected back into 3-D Euclidean space. The result is shown in Fig. 2(c). We see how each fiber tract is segmented completely without influence from the other crossing fiber tract.

\section{B. Real Data}

1) Material: The diffusion images were obtained on a healthy volunteer with a 3T MRI scanner. We used a diffusion weighted single shot EPI sequence with timing parameters: $\mathrm{TR} / \mathrm{TE} / \Delta / \delta=3000 / 154 / 47.6 / 35 \mathrm{~ms}, b_{\max }=12000 \mathrm{~mm}^{2} / \mathrm{s}$ and a spatial resolution of $2 \times 2 \times 3 \mathrm{~mm}^{3}$. The axial acquisition matrix was $128 \times 128$ and the slice number equal to 32 . The data were acquired by sampling 515 brain volumes, each with a different diffusion weighting corresponding to a different point in q-space. The points in q-space are restricted to the interior of a sphere where the maximal radius corresponds to $b_{\max }$. From this acquisition the ODF map is reconstructed according a standard DSI scheme [35]. A visual inspection was made in order to make a first quality estimation of the data. The subject has been lying sufficiently still so that no major artifact

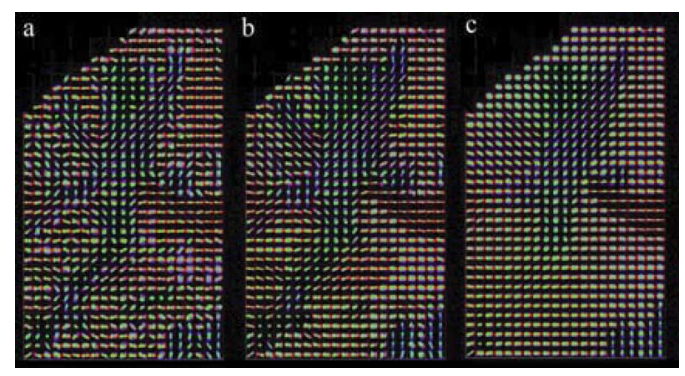

Fig. 5. A 2-D section of the 3-D space of the ODF volumes at different scales. (a) No smoothing applied. (b) Smoothed with a TV-flow, five iterations. (c) Ten iterations.

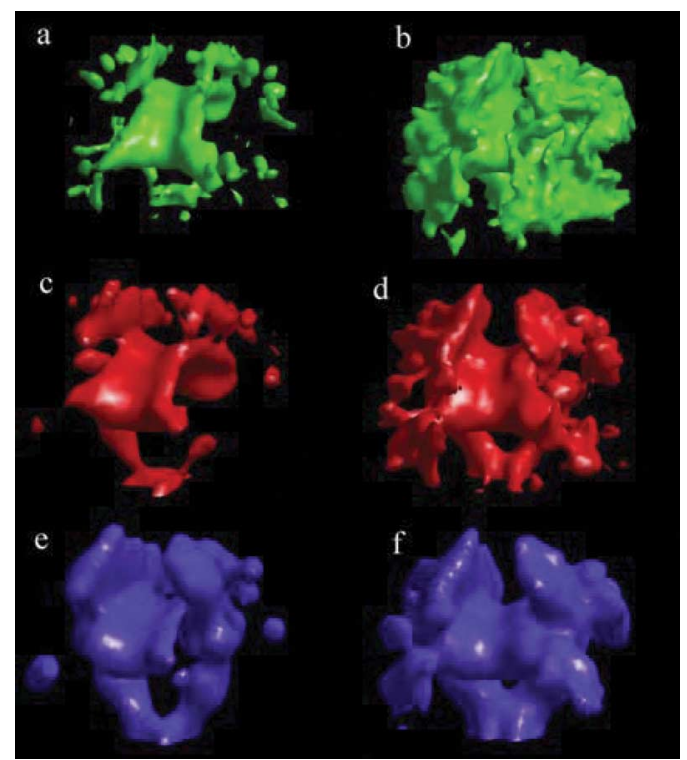

Fig. 6. Projections back to 3-D of different thresholds applied in 5-D space of different smoothing scales. (a) No smoothing applied, threshold $t=0.82$. (b) No smoothing applied, $t=0.75$. (c) Image smoothed with TV flow, five iterations, $t=0.60$. (d) TV-flow five iterations, $t=0.55$. (e) TV-flow ten iterations, $t=0.40$. (f) TV-flow ten iterations, $t=0.35$.

can be seen. Motion artifact due to cardiac and breathing are random effects that get averaged out increasing noise to a yet acceptable level. Informed consent was obtained in accordance with institutional guidelines for the volunteer.

2) Results: The ODFs are normalized by dividing with the maximum from each ODF and the 5-D POS is constructed with a sampling step of $n=18$ in orientation, (21), which corresponds to an angular step of $10^{\circ}$. The TV flow is then applied. In Fig. 5, the results can be seen after five and ten iterations, respectively. The figure shows the reconstructed ODF map after the regularization. The effect of the regularization can be easily seen in Fig. 6, where different thresholds have been applied to the level set function and the result of the thresholding has been projected back to three dimensions. We see that as the regularization gets more important the shape of the surfaces is simplified and the larger tract more distinctive as the smaller tracts are erased.

In order to place the initial surfaces the thresholding was performed on the regularized image. By only keeping data for certain angles the core shape of the corpus callosum, the cortico 


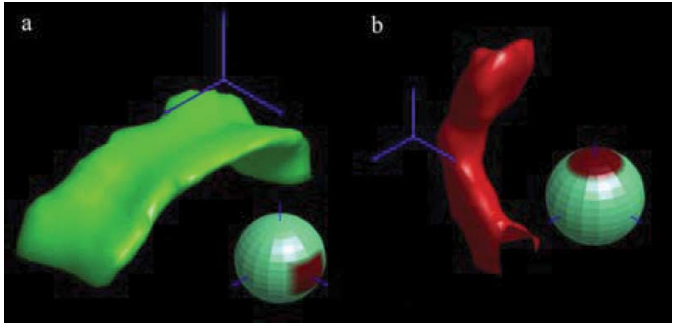

Fig. 7. Initial surfaces for the segmentation. Surfaces are obtained by thresholding the regularized 5-D space. In the 5-D space, only angles represented by red on the spheres are kept.

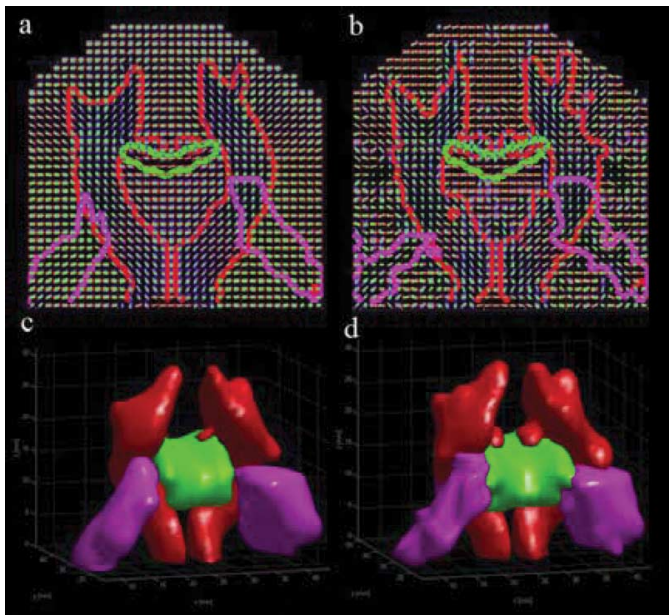

Fig. 8. Results from application on DSI from a human brain. Cortico spinal tract is represented by the red surface, the green surface represents the corpus callosum, and the magenta are the longitudinal fasciculus. (a) Coronal cut of the 3-D projection of the result superposed on a coronal slice of the regularized ODFs. (b) Coronal cut of the 3-D projection of the result superposed on a coronal slice of the original ODFs. (c) The 3-D projection of the 5-D results in a 5-D field regularized with ten iterations of the TV-flow. (d) The 3-D projection of the 5-D results in the original unregularized 5-D field.

spinal tracts and a part of the fasciculus bundles could easily be identified; see Fig. 7.

The initial surfaces were then evolved according to the method presented. The segmentation was first done in the lowest scale, that is the regularized image seen in Fig. 5(c), to obtain the global structure of the object. This results in a coarse segmentation as can be seen in Fig. 8(a) and (c). The refinement was then done by using the coarser segmentation as initialization and then evolving the surfaces with the previous method in the original 5-D POS. The 3-D projections of the results are shown in Fig. 8(b) and (d). They display the core of important fiber tracts such as the corpus callosum (green), the cortico spinal tract (red), and the arcuate fasciculus (magenta).

\section{Discussion AND CONCLUSION}

With this study, we have first of all practically and theoretically shown that it is possible to implement the level set method for evolving a hyper-surface in a high-dimensional non-Euclidean space. In particular, we have seen that the mean-curvature flow can be elegantly implemented by the use of the theory developed by Ambrosio and Soner [28] and that the segmentation process can be made more efficient with a multiscale approach. Second, we have seen that it is natural to represent diffusion of a complex medium with a 5-D space and that it has the additional advantage to have a mathematically clear representation. Furthermore, the dimensionality extension from 3-D to 5-D has the great advantage of disentangling signals coming from crossing structures. Results on synthetic data show that crossing fiber tracts in 3-D are represented in 5-D POS as separate objects characterized by intense diffusion. The results shown for brain DSI data clearly demonstrate the potential of this approach to clearly delimit fiber tracts, precisely structures of intense coherent diffusion. Such objects may then easily be characterized in terms of shape, volume, and internal signal characteristics, which is not so simple in standard tractography. From a signal processing point of view, the most attractive aspect of modelling diffusion as a scalar field on a high-dimensional manifold is that standard image processing procedures, such the ones used in 2-D and 3-D image processing, can be directly extended and adapted to explore dMRI data.

However, we must acknowledge that the presented procedure has also drawbacks as compared to standard tractography methods. Segmentation methods as presented here identify regions but are not able to measure connectivity between gray matter regions as done with tractography. The procedure is based on an augmentation of the dimensionality of the processing space. This generates a well known drawback, namely the "curse of dimensionality" [36] which refers to the exponential extension of the space as the number of dimensions increase, making the computations heavier. Thus, this new representation is a tradeoff between the additional computational weight and the greater conceptual simplicity. But we must keep in mind that the problem of data handling will be solved with the ever increasing computer power and a more efficient implementation and data storage.

It is not only interesting to compare the presented procedure with standard tractography as just discussed, but also to understand the differences and similarities between the presented level set method which is based on continuous mathematics with the Markovian approach which is discrete and developed in the context of dMRI in [16]. Both techniques are very well studied methods with solid backgrounds but differ significantly in their approaches [19], [20], [37]. The first technique considers the segmentation space to be a (continuous) manifold while for the second it is a (discrete) graph. The signal on the space is also considered to be of a different nature, once it is a continuous deterministic function and once it is a realization of a stochastic process, i.e., a random field. Both techniques have regularization constraints, once it is formulated in terms of curvature and once in terms of Gibbsian enery. Finally, the segmentation result is provided by the level set itself for the first approach and by the hidden Markov random field for the second. We see that both approaches differ by their theoretical foundations but perform the same tasks, namely regularization and segmentation. It is worth noticing that unifying theory of these two approaches is discussed in the scientific community [38], but is out of the scope of this paper, so is a quantitative comparison between their performances.

As already mentioned, our approach treats one fiber tract as one single object characterized by intense and coherent diffu- 
sion. This representation gives a different view of the brain architecture that can be more appropriate for applications such as quantitative investigation of the diffusion as well as for surgical planning and white matter registration. Quantitative measurements, like fractional anisotropy and mean diffusion, may be indicated in specific tracts for diseases like Wallerian degeneration [39], multiple sclerosis [40], schizophrenia [41], or for brain maturation studies [42].

\section{ACKNOWLEDGMENT}

The authors would like to thank R. Meuli and P. Schnyder for their support.

\section{REFERENCES}

[1] P. Basser, J. Mattiello, and D. Le Bihan, "MR diffusion tensor spectography and imaging," Biophys. J., vol. 66, pp. 259-267, 1994.

[2] P. Basser, J. Mattiello, and D. LeBihan, "Estimation of the effective self-diffusion tensor from the NMR spin echo," J. Magn. Reson., vol. 103, pp. 247-254, 1994.

[3] P. Hagmann, L. Jonasson, P. Maeder, J.-P. Thiran, V. Wedeen, and R. Meuli, "Understanding diffusion MR imaging techniques: From scalar diffusion-weighted imaging to diffusion tensor imaging and beyond," Radiographics, vol. 26, pp. S205-S223, 2006.

[4] V. Wedeen, T. Reese, D. Tuch, M. R. Wiegell, T. Weisskoff, and D. Chessler, "Mapping fiber orientation spectra in cerebral white matter with Fourier-transform diffusion MRI," in Proc. 8th Annu. Meeting ISMRM, Denver, CO, 2000, pp. 82-182.

[5] V. Wedeen, P. Hagmann, W. Tseng, T. Reese, and R. Weisskoff, "Mapping complex tissue architecture with diffusion spectrum magnetic resonance imaging," Magn. Reson. Med., vol. 54, no. 6, pp. 1377-1386, 2005.

[6] P. Callaghan, Principles of Nuclear Magnetic Resonance Microscopy. Oxford, U.K.: Oxford Univ. Press, 1991.

[7] D. Cory and A. Garroway, "Measurement of translational displacement probabilies by NMR: An indicator of compartmentation," Magn. Reson. Med., vol. 14, pp. 435-444, 1990.

[8] P. Hagmann, T. Reese, W. Tseng, R. Meuli, J. Thiran, and V. Wedeen, "Diffusion spectrum imaging tractography in complex cerebral white matter: An investigation of the centrum semiovale," in Proc. ISMRM 12th Sci. Meeting, May 2004, vol. 12, p. 623.

[9] D. Tuch, T. Reese, M. Wiegell, and V. Wedeen, "Diffusion MRI of complex neural architecture," Neuron, vol. 40, no. 5, pp. 885-895, Dec. 4, 2003.

[10] J. Tournier, F. Calamante, D. Gadian, and A. Connelly, "Direct estimation of the fiber orientation density function from diffusion-weighted MRI data using spherical deconvolution," NeuroImage, vol. 3, pp. $1176-1185,2004$

[11] K. Jansons and D. Alexander, "Persistent angular structure: New insights from diffusion MRI data," Inverse Problems, vol. 19, pp. 1031-1046, 2003.

[12] P. Hagmann, L. Cammoun, R. Martuzzi, P. Maeder, S. Clarke, J. Thiran, and R. Meuli, "Hand preference and sex shape the architecture of language networks," Hum. Brain Mapp., vol. 27, no. 10, pp. 828-835, 2006.

[13] P. Hagmann, "From diffusion MRI to brain connectomics," Ph.D. dissertation, Ecole Polytechnique Federale de Lausanne (EPFL), Lausanne, Switzerland, 2005.

[14] P. Hagmann, M. Kurant, X. Gigandet, P. Thiran, V. Wedeen, R. Meuli, and J.-P. Thiran, "Imaging the brain neuronal network with diffusion MRI: A way to understand its global architecture," in Proc. Int. Soc. Magn. Reson. Med., 2006, pp. 436-1436.

[15] L. Jonasson, X. Bresson, P. Hagmann, R. Meuli, O. Cuisenaire, and J.-P. Thiran, "White matter fiber tract segmentation in DT-MRI using geometric flows," Med. Image Anal., vol. 9, no. 3, pp. 223-236, Jun. 2005.

[16] P. Hagmann, L. Jonasson, T. Deffieux, R. Meuli, J. Thiran, and V. Wedeen, "Fibertract segmentation in position orientation space from high angular resolution diffusion MRI," NeuroImage, vol. 32, no. 2, pp. 665-675, 2006.
[17] T. Chan and L. A. Vese, "Active contours without edges," IEEE Trans. Image Process., vol. 10, no. 2, pp. 266-277, Feb. 2001.

[18] S. Osher and J. Sethian, "Fronts propagating with curvature-dependent speed: Algorithms based on hamilton-jacobi formulations," J. Comput. Phys., vol. 79, pp. 12-49, 1988.

[19] S. Osher, Geometric Level Set Method in Imaging, Vision and Graphics. Berlin, Germany: Springer-Verlag, 2003.

[20] J. Sethian, Level Set Methods and Fast Marching Methods: Evolving Interfaces in Computational Geometry, Fluid Mechanics, Computer Vision, and Materials Science. Cambridge, U.K.: Cambridge Univ. Press, 1999.

[21] L. Rudin, S. Osher, and E. Fatemi, "Nonlinear total variation based noise removal algorithms," Physica D, vol. 60, no. 1-4, pp. 259-268, 1992.

[22] F. Andreu, C. Ballester, V. Caselles, and J. M. Mazon, "Minimizing total variation flow," Different. Integr. Equat., vol. 14, no. 3, pp. 321-360, 2001.

[23] D. Tuch, "Q-ball imaging," Magn. Reson. Med., vol. 52, no. 6, pp. 1358-1372, Dec. 2004.

[24] E. Kreyszig, Differential Geometry, . New York: Dover, 1991.

[25] V. Caselles, R. Kimmel, and G. Sapiro, "Geodesic active contours," Int. J. Comput. Vis., vol. 22, pp. 61-79, 1997.

[26] S. Kichenassamy, A. Kumar, P. Olver, A. Tannenbaum, and A. Yezzi, "Conformal curvature flows: From phase transitions to active vision," Arch. Ration. Mech. Anal., vol. 134, pp. 275-301, 1996.

[27] V. Caselles, R. Kimmel, G. Sapiro, and C. Sbert, "Minimal surfaces: A geometric three dimensional segmentation approach," Numer. Math., vol. 77, no. 4, pp. 423-425, Oct. 1, 1997.

[28] L. Ambrosio and H. Soner, "Level set approach to mean curvature flow in arbitrary codimension," J. Differ. Geom., vol. 43, pp. 693-737, 1996.

[29] Y. Chen, Y. Giga, and S. Goto, "Uniqueness and existence of viscosity solutions of generalized mean curvature flow equations," J. Differ. Geom., vol. 33, pp. 749-786, 1991.

[30] D. Mumford and J. Shah, "Optimal approximation by piecewise smooth functions and associated variational problems," Commun. Pure Appl. Math., vol. 42, pp. 577-685, 1989.

[31] B. Leroy, I. Herlin, and L. Cohen, "Multi-resolution algorithms for active contour models," in Proc. 12th Int. Conf. Anal. Optim. Syst., 1996, pp. 58-65.

[32] A. Petrovic, O. Divorra Escoda, and P. Vandergheynst, "Multiresolution segmentation of natural images: From linear to nonlinear scalespace representations," IEEE Trans. Image Process., vol. 13, no. 8, pp. 1104-1114, Aug. 2004.

[33] J. Weickert, "A review of nonlinear diffusion filtering," in Scale-Space Theory in Computer Vision. : Springer-Verlag, 1997, Lecture Notes in Computer Science.

[34] D. Adalsteinsson and J. Sethian, "A fast level set method for propagating interfaces," J. Comput. Phys., vol. 118, no. 2, pp. 269-277, May. 1995.

[35] V. Wedeen, P. Hagmann, W. Tseng, T. Reese, and T. Weisskoff, "Mapping complex tissue architecture with diffusion spectrum magnetic resonance imaging," Magn. Res Med., vol. 54, pp. 1377-1386, 2005.

[36] R. Bellman, Adaptive Control Processes: A Guided Tour. Princeton, NJ: Princeton Univ. Press, 1961.

[37] D. Geman, Random-fields and inverse problems in imaging. Berlin, Germany: Springer-Verlag, 1990, vol. 1427, Lecture Notes in Mathematics, pp. 113-193.

[38] X. Bresson, "Image segmentation with variational active contours," Ph.D. dissertation, EPFL, Lausanne, Switzerland, 2005.

[39] C. Pierpaoli, A. Barnett, S. Pajevic, R. Chen, L. Penix, A. Virta, and P. Basser, "Water diffusion changes in wallerian degeneration and their dependence on white matter architecture," Neurolmage, vol. 13, no. 6, pp. 1174-1185, Jun. 2001.

[40] M. Filippi, M. Cercignani, M. Inglese, M. Horsfield, and G. Comi, "Diffusion tensor magnetic resonance imaging in multiple sclerosis," Neurology, vol. 56, no. 3, pp. 304-311, 2001.

[41] M. Kubicki, H. Park, C. Westin, P. Nestor, R. Mulkern, S. Maier, M. Niznikiewicz, E. Connor, J. Levitt, and M. Fruminothers, "DTI and MTR abnormalities in schizophrenia: Analysis of white matter integrity," NeuroImage, vol. 26, no. 4, pp. 1109-1118, 2005.

[42] P. Huppi, S. Warfield, R. Kikinis, P. Barnes, G. Zientara, F. Jolesz, M. Tsuji, and J. Volpe, "Quantitative magnetic resonance imaging of brain development in premature and mature newborns," Ann. Neurol., vol. 43, no. 2, pp. 224-235, Feb. 1998. 